\title{
Erratum
}

\section{A revenue management slot allocation model for liner shipping networks}

Sebastian Zurheide and Kathrin Fischer

Maritime Economics \& Logistics (2013) 15, 523. doi:10.1057/mel.2013.6

Correction to: Maritime Economics \& Logistics (2012), Vol. 14, 3, 344-361. doi:10.1057/mel.2012.11

In the above paper constraint (3) was incorrect. Please see the correct version of constraint (3) below.

$$
\begin{array}{r}
\sum_{t \in T Y} \sum_{\begin{array}{c}
b_{k_{h}} \in P A_{k} \\
\text { with } k \in S V \\
\text { and } h \in B C_{k}
\end{array}} Y_{(o, d)_{k_{i}}}^{B} b_{k_{h}}\left(W_{t b_{k_{h}}}^{F} \sum_{S \in S E} x_{s t b_{k_{h}}}^{F}+W_{t}^{E} x_{t b_{k_{h}}}^{E}\right) \leq D W_{(o, d)_{k_{i}}}, \\
\forall(o, d)_{k_{i}} \in L E_{k} \text { with } k \in S V \text { and } i \in S C_{k}
\end{array}
$$

